\title{
MICROCOMPUTERS IN AN INTRODUCTORY COLLEGE ASTRONOMY LABORATORY: A SOFTWARE DEVELOPMENT PROJECT
}

\author{
David D. Meisel ${ }^{1}$, Kenneth F. Kinsey, and Charles H. Recchia \\ Department of Physics and Astronomy, State University College at Geneseo, \\ Geneseo, New York 14454 U.S.A.
}

We have developed software for the Apple Ile series of microcomputers for use in labs in an introductory astronomy course. This software emphasizes a toolkit approach to data analysis; it has been class tested with over 170 students and was a resounding success as a replacement for previously used graphical approximations. A unique feature of this software is the incorporation of image-processing techniques into a course designed for non-science majors.

The five software packages are:

(a) Datasheet - A six-column spreadsheet with columnwise operations, statistical functions, and double-high-resolution graphics.

(b) Image-Processor Program - Allows $37 \times 27$ pixel $\times 8$ bit video captured images to be manipulated using standard image-processing techniques such as low pass/high pass filtering and histogram equalization.

(c) Picture-Processor Program - Allows $256 \times 192$ bilevel pictures to be manipulated and measured with functions that include calipers, odometer, planimeter, and protractor.

(d) Orrery Program - Simulates planet configurations along the ecliptic. A movable cursor allows selection of specific configurations. Since both relative times and angular positions are given, students can deduce the scale of the solar system using simple trigonometry.

(e) Plot Program - Allows orbital positions as observed from above the pole to be plotted on the screen. By entering trial values of elliptical orbit parameters, students obtain and the program plots the best fitting ellipse to the data. The sum of the squares of the residuals in the radial coordinate is given after each trial so that students can discover convergence more easily than by simple visual examination of a plot comparing the trial theoretical points with the raw data points.

The video-captured materials used with the Image Processing and Picture Processing programs were obtained first on VHS video tape using the $0.6-\mathrm{m}$ telescope at the C.E.K. Mees Observatory (near Bristol Springs, New York State) or the $0.35-\mathrm{m}$ telescope at SUNY/Geneseo. Selected video frames were subsequently captured into

\footnotetext{
${ }^{1}$ Also Associate Director of the C.E.K. Mees Observatory, University of Rochester
} 
$256 \times 192$ pixels $\mathrm{x} 8$ bit video images using the Imageworks ${ }^{T M}$ system (Redshift Ltd., P.O. Box 4335, Mountain View, California, 94040) on an Apple Ile and recorded to floppy disk. Using our own extensively modified version of the Imageworks ${ }^{T M}$ software, we were able to create images and bilevel pictures that can be loaded directly into any Apple Ile for processing. Images presently available include selected lunar features, Jupiter, Saturn, Jovian satellites, Venus, double stars, solar activity, and low dispersion stellar spectra. We are striving to expand this list to other objects. Astronomers having public-domain images they might be willing to share should write to us giving details.

Our approach to computer usage is (1) to provide menu-driven programs that furnish tools that are analogous to those used in classical laboratory tasks; (2) to give students as much control as possible over the order in which the tasks are done; (3) to restrict computer use to those tasks where it is essential or has greatest impact; (4) to provide a consistent interface to more advanced features that will be used in later courses; and (5) to provide students with their own copies of software and images, at the price of a diskette, to allow work outside the laboratory.

In summary, we have successfully brought low-cost image processing techniques into elementary astronomy laboratories through an extension of a toolkit programming approach. Our materials are all in the public-domain and therefore available at minimum cost. The software runs under the PRODOS system, and has been designed for the 128k Apple IIe (enhanced) series, and is being extended to the Apple IIgs model.

Distribution copies including ASCII readable notes are now available for: DATASHEET 6.0 (one 5 1/4" disk); PICTURE/IMAGE (one 5 1/4" disk); ORRERY/PLOT (one $51 / 4$ " disk); Imageworks ${ }^{T M}$ Enhanced Software (one $51 / 4$ " disk)*; Imageworks ${ }^{T M}$ Compatible Images (one $51 / 4 "$ disk) ${ }^{*}$.

Requests for these should be accompanied by the appropriate type and number of blank diskettes. Requestors will be put on a mailing list for notices of updates. Address all inquiries to: Dr. David D. Meisel, Department of Physics and Astronomy, State University College, Geneseo, New York 14454, U.S.A.. Other inquiries can be made by mail or BITNET (MEISEL@GENESEO).

${ }^{*}$ Requires Imageworks ${ }^{T M}$ board to function properly. Xerox copy of user manual should accompany request. 